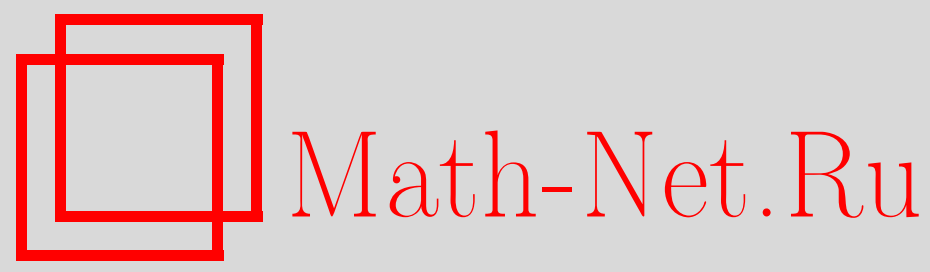

Г. Л. Кулинич, Ю. В. Бернацкая, О фазовом портрете гармонического осциллятора с трением, возмущенного случайным процессом типа белого шума, Матем. заметки, 2000, том 68, выпуск 6, 862-869

DOI: https://doi.org/10.4213/mzm1009

Использование Общероссийского математического портала Math-Net.Ru подразумевает, что вы прочитали и согласны с пользовательским соглашением http://www.mathnet.ru/rus/agreement

Параметры загрузки:

IP: 3.82 .47 .9

26 апреля 2023 г., 18:04:02

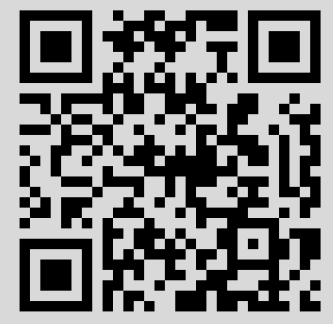




\title{
О ФАЗОВОМ ПОРТРЕТЕ ГАРМОНИЧЕСКОГО ОСЦИЛЛЯТОРА С ТРЕНИЕМ, ВОЗМУЩЕННОГО СЛУЧАЙНЫМ ПРОЦЕССОМ ТИПА БЕЛОГО ШУМА
}

\author{
Г. Л. Кулинич, Ю. В. Бернацкая
}

Исследуется фазовый портрет гармонического осциллятора с трением в случае, когда под определенным углом к вектору фазовой скорости осциллятора воздействуют случайные возмущения типа белого шума в форме Ито.

Библиография: 10 названий.

Гармоническим осииллятором с трением называют систему, движение которой описьвается линейньм дифференциальным уравнением второго порядка

$$
\ddot{u}(t)+2 h \dot{u}(t)+k^{2} u(t)=0, \quad u(0)=u_{0}, \quad \dot{u}(0)=\dot{u}_{0},
$$

где $u_{0}, \dot{u}_{0}$ - начальные положение и скорость осциллятора, $u_{0}^{2}+\dot{u}_{0}^{2}>0$; постоянные $k>0, h$ - параметры осциллятора ( $2 h$ - коэффициент трения); $u(t), \dot{u}(t)$ - положение и скорость осциллятора в момент времени $t>0$.

Уравнение (1) эквивалентно системе дифференциальных уравнений первого порядка

$$
\dot{x}(t)=B x(t), \quad B=\left(\begin{array}{cc}
0 & 1 \\
-k^{2} & -2 h
\end{array}\right), \quad x(t)=\left(\begin{array}{c}
x_{1}(t) \\
x_{2}(t)
\end{array}\right),
$$

где $x_{1}(t)=u(t), x_{2}(t)=\dot{u}(t)$.

В прямоугольной декартовой системе координат $X_{1} O X_{2}$ состояние гармонического осциллятора изображается точкой $M$ с координатами $\left(x_{1}(t), x_{2}(t)\right)$, которая движется по фазовой траектории, а фазовая скорость изображается вектором $\left(x_{2}(t),-k^{2} x_{1}(t)-\right.$ $\left.2 h x_{2}(t)\right)$, которьй направлен по касательной к фазовой траектории в точке $M$.

Портрет возможных движений точки $M$ на фазовой плоскости $X_{1} O X_{2}$ зависит от знака величины $\left(h^{2}-k^{2}\right)$ (при $0<h^{2}<k^{2}$ - семейство спиралей, при $h^{2}>k^{2}-$ семейство “парабол", при $h^{2}=k^{2}$ - семейство кривых параболического типа) [1].

В данной заметке исследуется поведение изображаюшей точки $M$ на фазовой плоскости $X_{1} O X_{2}$ системы (2), возмущенной флуктуациями типа белого шума в форме Ито, которые воздействуют на систему под определенньм углом к вектору фазовой скорости, т. е. для классов

$$
K_{1}:|h|<k ; \quad K_{2}:|h|>k ; \quad K_{3}:|h|=k
$$

исследуется система стохастических дифференциальных уравнений Ито

$$
d x(t)=d \zeta(t) x(t), \quad x_{1}(0)=u_{0}, \quad x_{2}(0)=\dot{u}_{0},
$$


где

$$
\zeta(t)=\int_{0}^{t} B_{q(s)} d s+\int_{0}^{t} B_{g(s)} d w(s),
$$

$B_{q}, B_{g}$ - матрицы вида:

1) для $K_{1}$

$$
B_{f}=\left(\begin{array}{cc}
f_{1}+\frac{h}{\mu} f_{2} & \frac{f_{2}}{\mu} \\
-\frac{k^{2}}{\mu} f_{2} & f_{1}-\frac{h}{\mu} f_{2}
\end{array}\right), \quad \mu=\sqrt{k^{2}-h^{2}}
$$

2) для $K_{2}$

$$
\begin{gathered}
B_{f}=\left(\begin{array}{cc}
\frac{1}{\lambda_{2}-\lambda_{1}}\left[\lambda_{2} f_{1}-\lambda_{1} f_{2}\right] & \frac{1}{\lambda_{2}-\lambda_{1}}\left[f_{2}-f_{1}\right] \\
-\lambda_{2} f_{1}+\frac{\lambda_{2}}{\lambda_{2}-\lambda_{1}}\left[\lambda_{2} f_{1}-\lambda_{1} f_{2}\right] & f_{1}+\frac{\lambda_{2}}{\lambda_{2}-\lambda_{1}}\left[f_{2}-f_{1}\right]
\end{array}\right), \\
\lambda_{1}=-h+\sqrt{h^{2}-k^{2}}, \quad \lambda_{2}=-h-\sqrt{h^{2}-k^{2}}
\end{gathered}
$$

3) для $K_{3}$

$$
B_{f}=\left(\begin{array}{cc}
f_{1}+h f_{2} & f_{2} \\
-h^{2} f_{2} & f_{1}-h f_{2}
\end{array}\right)
$$

при $f_{i}=q_{i}(t), f_{i}=g_{i}(t)$ соответственно; $\left(w(t), \mathscr{F}_{t}\right)$ - одномерньй винеровский процесс, $\mathscr{F}_{t}$ - неубывающий поток $\sigma$-алгебр на фиксированном вероятностном пространстве $(\Omega, \mathscr{F}, P) ; q_{i}(t), g_{i}(t)$ - случайные локально ограниченные функции, при каждом $t \geqslant 0$ измеримые относительно $\mathscr{F}_{t}$. Для уравнения (3) выписан явный вид решения (теорема 1); получены необходимые и достаточные условия, при которых изображающая точка $M$ с вероятностью 1 “диффундирует” по определенным гладким кривьм (теорема 2). Для класса $K_{1}$ приведены достаточные условия асимптотической устойчивости и неустойчивости положения равновесия системы (3), при этом исследуется поведение угловой характеристики решения (теорема 3 ).

Исследование поведения изображающей точки $M$ системы (3) и поведения корреляционной функции решения при $q_{1} \equiv 0, g_{1} \equiv 0$ (возмушение системы (2) вдоль вектора фазовой скорости) проведено при $h \neq 0$ в работе [2], а при $h=0$ - в работе [3], при этом в работе [3] рассматривается бельй шум в форме Ито и в форме Стратоновича. В работе [4] для линейных систем вида (3) в $\mathbb{R}^{n}$ с переменными неслучайными коэффициентами выписана формула для нахождения фундаментальной матрищы уравнения, а с постоянньми коэффициентами - исследуются условия устойчивости и неустойчивости решений, при этом необходимые и достаточные условия устойчивости выражаются через эргодическую меру для процесса $x(t)|x(t)|^{-1}$. Модели гармонического осциллятора при случайных возмущениях лишь второй компоненты фазовой скорости исследовались в целом ряде работ (например [5]-[8]).

Перейдем к изложению результатов.

Теорема 1. Пусть $x(t)$ - решение уравнения (3). Тогда с вероятностью 1 при всех $t \geqslant 0$ имеют место равенства:

1) $\left.\partial \Omega_{\text {g }} K_{1}\left(x_{1}(0) \neq 0\right)\right)$

$$
x_{1}(t)=A_{1}(t) \cos \varphi_{1}(t), \quad x_{2}(t)=k A_{1}(t) \sin \left(\varphi_{1}(t)+\gamma_{1}\right),
$$


әде

$$
\begin{gathered}
A_{1}(t)=\left[x_{1}^{2}(0)+\left(\frac{h x_{1}(0)+x_{2}(0)}{\mu}\right)^{2}\right]^{1 / 2} \exp \left\{\int_{0}^{t}\left(q_{1}(s)+\frac{1}{2}\left[g_{2}^{2}(s)-g_{1}^{2}(s)\right]\right) d s\right. \\
\left.+\int_{0}^{t} g_{1}(s) d w(s)\right\} \\
\varphi_{1}(t)=\varphi_{1}(0)+\int_{0}^{t}\left[g_{1}(s) g_{2}(s)-q_{2}(s)\right] d s-\int_{0}^{t} g_{2}(s) d w(s) \\
\operatorname{tg} \varphi_{1}(0)=\frac{h x_{1}(0)+x_{2}(0)}{\mu x_{1}(0)}, \quad \operatorname{tg} \gamma_{1}=-\frac{h}{\mu}
\end{gathered}
$$

2) $\partial$ л $K_{2}\left(-\lambda_{2} x_{1}(0)+x_{2}(0) \neq 0\right)$

$x_{1}(t)=A_{2}(t) \sqrt{2} \cos \varphi_{2}(t), \quad x_{2}(t)=A_{2}(t)\left(\lambda_{1}^{2}+\lambda_{2}^{2}\right)^{1 / 2} \sin \left(\varphi_{2}(t)+\gamma_{2}\right)$,

$2 \partial e$

$$
\begin{gathered}
A_{2}(t)=\frac{1}{\lambda_{1}-\lambda_{2}}\left[y_{1}^{2}(t)+y_{2}^{2}(t)\right]^{1 / 2}, \\
y_{i}(t)=y_{i}(0) \exp \left\{\int_{0}^{t}\left[q_{i}(s)-\frac{1}{2} g_{i}^{2}(s)\right] d s+\int_{0}^{t} g_{i}(s) d w(s)\right\}, \quad i=1,2, \\
y_{1}(0)=-\lambda_{2} x_{1}(0)+x_{2}(0), \quad y_{2}(0)=-\lambda_{1} x_{1}(0)+x_{2}(0), \\
\operatorname{tg} \varphi_{2}(t)=\frac{y_{2}(t)}{y_{1}(t)}, \quad \operatorname{tg} \gamma_{2}=\frac{-\lambda_{1}}{\lambda_{2}}
\end{gathered}
$$

3) для $K_{3}\left(h x_{1}(0)+x_{2}(0) \neq 0\right)$

$$
x_{1}(t)=A_{3}(t) \sin \varphi_{3}(t), \quad x_{2}(t)=A_{3}(t) \sqrt{1+h^{2}} \cos \left(\varphi_{3}(t)+\gamma_{3}\right),
$$

$2 \partial e$

$$
\begin{gathered}
A_{3}(t)=\exp \left\{\int_{0}^{t}\left[q_{1}(s)-\frac{1}{2} g_{1}^{2}(s)\right] d s+\int_{0}^{t} g_{1}(s) d w(s)\right\}\left\{\left(h x_{1}(0)+x_{2}(0)\right)^{2}\right. \\
\left.+\left[x_{1}(0)+\left(h x_{1}(0)+x_{2}(0)\right)\left(\int_{0}^{t}\left[q_{2}(s)-g_{1}(s) g_{2}(s)\right] d s+\int_{0}^{t} g_{2}(s) d w(s)\right)\right]^{2}\right\}^{1 / 2}, \\
\operatorname{tg} \varphi_{3}(t)=\frac{x_{1}(0)}{h x_{1}(0)+x_{2}(0)}+\int_{0}^{t}\left[q_{2}(s)-g_{1}(s) g_{2}(s)\right] d s+\int_{0}^{t} g_{2}(s) d w(s), \\
\operatorname{tg} \gamma_{3}=h .
\end{gathered}
$$


ДокАЗАТЕЛЬСтво теоремы для $K_{1}$. Рассмотрим линейноепреобразованиепроцесса $x(t)$, при котором матрица $B_{f}$ в уравнении (3) примет жордановую форму, т. е. процесс $y(t)=T x(t)$, где

$$
T=\left(\begin{array}{ll}
\mu & 0 \\
h & 1
\end{array}\right) \quad\left(T B_{f} T^{-1}=\left(\begin{array}{cc}
f_{1} & f_{2} \\
-f_{2} & f_{1}
\end{array}\right)\right) .
$$

Согласно уравнению (3) для процесса $y(t)$ имеем стохастическое дифференциальное уравнение

$$
d y(t)=T d \zeta(t) T^{-1} y(t) .
$$

Введем новые процессы $r(t), \varphi(t)$ через соотношения

$$
y_{1}(t)=r(t) \cos \varphi(t), \quad y_{2}(t)=r(t) \sin \varphi(t) .
$$

Тогда [3]

$$
\left\{\begin{array}{l}
d r(t)=r(t)\left(\left[q_{1}(t)+\frac{1}{2} g_{2}^{2}(t)\right] d t+g_{1}(t) d w(t)\right) \\
d \varphi(t)=\left(-q_{2}(t)+g_{1}(t) g_{2}(t)\right) d t-g_{2}(t) d w(t)
\end{array}\right.
$$

с вероятностью 1 для всех $t \geqslant 0$.

Поэтому [9]

$$
\left\{\begin{array}{l}
r(t)=r(0) \exp \left\{\int_{0}^{t}\left(q_{1}(s)+\frac{1}{2}\left[g_{2}^{2}(s)-g_{1}^{2}(s)\right]\right) d s+\int_{0}^{t} g_{1}(s) d w(s)\right\}, \\
\varphi(t)=\varphi(0)-\int_{0}^{t}\left(q_{2}(s)-g_{1}(s)-g_{2}(s)\right) d s-\int_{0}^{t} g_{2}(s) d w(s) .
\end{array}\right.
$$

Поскольку $x(t)=T^{-1} y(t)$, где $T^{-1}-$ матрища, обратная к $T$, то, учитывая явньй вид процесса $y(t)$, получим доказательство теоремы для класса $K_{1}$.

Доказательство теоремы для $K_{2}, K_{3}$ аналогично доказательству для $K_{1}$ с тем лишь отличием, что здесь используются матрищы

$$
T=\left(\begin{array}{ll}
-\lambda_{2} & 1 \\
-\lambda_{1} & 1
\end{array}\right) \quad\left(T B_{f} T^{-1}=\left(\begin{array}{cc}
f_{1} & 0 \\
0 & f_{2}
\end{array}\right)\right)
$$

для $K_{2}$,

$$
T=\left(\begin{array}{cc}
h & 1 \\
1 & 0
\end{array}\right) \quad\left(T B_{f} T^{-1}=\left(\begin{array}{ll}
f_{1} & 0 \\
f_{2} & f_{1}
\end{array}\right)\right)
$$

для $K_{3}$ и находится явный вид решений уравнения (4). Для $K_{2}$ решение находится непосредственно [9]:

$$
\begin{gathered}
y_{i}(t)=y_{i}(0) \exp \left\{\int_{0}^{t}\left[q_{i}(s)-\frac{1}{2} g_{i}^{2}(s)\right] d s+\int_{0}^{t} g_{i}(s) d w(s)\right\}, \\
y_{1}(0)=-\lambda_{2} x_{1}(0)+x_{2}(0), \quad y_{2}(0)=-\lambda_{1} x_{1}(0)+x_{2}(0) .
\end{gathered}
$$


Для $K_{3}$ решение находится методом, предложенным в работе [10]:

$$
\begin{gathered}
y_{1}(t)=y_{1}(0) \exp \left\{\int_{0}^{t}\left[q_{1}(s)-\frac{1}{2} g_{1}^{2}(s)\right] d s+\int_{0}^{t} g_{1}(s) d w(s)\right\} \\
y_{2}(t)=\left[y_{2}(0) y_{1}^{-1}(0)+\int_{0}^{t}\left[q_{2}(s)-g_{1}(s) g_{2}(s)\right] d s+\int_{0}^{t} g_{2}(s) d w(s)\right] y_{1}(t), \\
y_{1}(0)=h x_{1}(0)+x_{2}(0), \quad y_{2}(0)=x_{1}(0) .
\end{gathered}
$$

Из явного вида решения уравнения (3) вытекает, что $x(t) \in D_{i}$ с вероятностью 1 для всех $t>0$ при условии, что $x(0) \in D_{i}$, где $D_{1}=\mathbb{R}^{2} \backslash\{0\}$ для $K_{1}, D_{2}=\left\{x:-\lambda_{1} x_{1}+x_{2}>0\right.$, $\left.-\lambda_{2} x_{1}+x_{2}>0\right\}$ для $K_{2}, D_{3}=\left\{x: h x_{1}+x_{2}>0\right\}$ для $K_{3}$. Поэтому исследование поведения решения уравнения (3) на фазовой плоскости $X_{1} O X_{2}$ достаточно провести соответственно в областях $D_{i}$. Для этого в областях $D_{i}$ рассмотрим кривые $G_{i}(x)=C$ :

$$
G_{1}(x)=r e^{-\alpha \varphi}
$$

где $(r, \varphi)$ - полярные координаты, при этом

$$
\begin{gathered}
x_{1}=r \mu^{-1} \cos \varphi, \quad x_{2}=r\left(\sin \varphi-h \mu^{-1} \cos \varphi\right) ; \\
G_{2}(x)=\left(-\lambda_{1} x_{1}+x_{2}\right)\left(-\lambda_{2} x_{1}+x_{2}\right)^{-\alpha}, \\
G_{3}(x)=x_{1}\left(h x_{1}+x_{2}\right)^{-1}-\alpha \ln \left(h x_{1}+x_{2}\right),
\end{gathered}
$$

и будем считать, что $x(0) \in D_{i}$. Тогда с вероятностью 1 для всех $t \geqslant 0$ соответственно имеют место равенства

$$
\begin{gathered}
G_{i}(x(t))=G_{i}(x(0)) \exp \left\{I_{i}(t)+J_{i}(t)\right\}, \quad i=1,2, \\
G_{3}(x(t))=G_{3}(x(0))+I_{3}(t)+J_{3}(t)
\end{gathered}
$$

где

$$
\begin{aligned}
& I_{1}(t)=\int_{0}^{t}\left(q_{1}(s)+\alpha q_{2}(s)+\frac{1}{2}\left[g_{2}^{2}(s)-g_{1}^{2}(s)\right]-\alpha g_{1}(s) g_{2}(s)\right) d s, \\
& \left.J_{1}(t)=\int_{0}^{t}\left(g_{1}(s)+\alpha g_{2}(s)\right) d w(s) \quad \text { (для } K_{1}\right), \\
& I_{2}(t)=\int_{0}^{t}\left(q_{2}(s)-\alpha q_{1}(s)-\frac{1}{2}\left[g_{2}^{2}(s)-\alpha g_{1}^{2}(s)\right]\right) d s, \\
& J_{2}(t)=\int_{0}^{t}\left(g_{2}(s)-\alpha g_{1}(s)\right) d w(s) \quad\left(\text { для } K_{2}\right), \\
& I_{3}(t)=\int_{0}^{t}\left(q_{2}(s)-\alpha q_{1}(s)-g_{1}(s) g_{2}(s)+\frac{\alpha}{2} g_{1}^{2}(s)\right) d s, \\
& \left.J_{3}(t)=\int_{0}^{t}\left(g_{2}(s)-\alpha g_{1}(s)\right) d w(s) \quad \text { (для } K_{3}\right) .
\end{aligned}
$$

Исходя из этих равенств, имеем следующую теорему. 
ТЕорема 2. Для того чтобы $G_{i}(x(t))=G_{i}(x(0))$ с вероятностью 1 для всех $t \geqslant 0$ соответственно для $K_{i}$, необходимо и достаточно, чтобб с вероятностью 1 почти для всех $t \geqslant 0$ имели место равенства

1) $\partial л я K_{1}$

$$
g_{1}(t)+\alpha g_{2}(t)=0, \quad q_{1}(t)+\alpha q_{2}(t)+\left(\frac{1+\alpha^{2}}{2}\right) g_{2}^{2}(t)=0
$$

2) $\partial л я K_{2}$

$$
g_{2}(t)-\alpha g_{1}(t)=0, \quad q_{2}(t)-\alpha q_{1}(t)+\frac{\alpha(1-\alpha)}{2} g_{1}^{2}(t)=0 ;
$$

3) $\partial л я K_{3}$

$$
g_{2}(t)-\alpha g_{1}(t)=0, \quad q_{2}(t)-\alpha q_{1}(t)-\frac{\alpha}{2} g_{1}^{2}(t)=0 .
$$

ДокАЗАТЕЛЬСТво. Исходя из соотношений (6), для равенства $G_{1}(x(t))=G_{1}(x(0))$ необходимо и достаточно, чтобы с вероятностью 1 для всех $t \geqslant 0$ вьполнялось равенство

$$
I_{1}(t)+J_{1}(t)=0 .
$$

Поскольку $I_{1}(t)$ с вероятностью 1 абсолютно непрерывная функция, а $J_{1}(t)$ - непрерывный с вероятностью 1 мартингал, то равенство (7) имеет место тогда и только тогда, когда $I_{1}(t)=0, J_{1}(t)=0$ с вероятностью 1 для всех $t \geqslant 0$. Поэтому для равенства (7) необходимо и достаточно, чтобы

$$
\begin{gathered}
g_{1}(t)+\alpha g_{2}(t)=0, \\
q_{1}(t)+\alpha q_{2}(t)-\alpha g_{1}(t) g_{2}(t)+\frac{1}{2}\left[g_{2}^{2}(t)-g_{1}^{2}(t)\right]=0
\end{gathered}
$$

с вероятностью 1 почти для всех $t \geqslant 0$. Из этих равенств вытекает утверждение теоремы для $K_{1}$. Аналогично получаем утверждение теоремы для $K_{2}, K_{3}$.

СлеДСтвиЕ 1. Для того чтобы фазовые траектории уравнения (2) соответственно для $K_{i}$ в областях $D_{i}$ были инвариантными кривыми уравнения (3), необходимо и достаточно выполнение условия теоремы 2 при $\alpha=h \mu^{-1} \partial л я K_{1}, n р и$ $\alpha=\lambda_{1} \lambda_{2}^{-1} \partial \Omega_{s} K_{2}$, при $\alpha=h^{-1} \partial \Omega_{s} K_{3}$.

Действительно, при указанных значениях $\alpha$ кривые $G_{i}(x)=C$ являются фазовыми траекториями уравнения (2) соответственно для $K_{i}$ в областях $D_{i}[1]$.

СледСтвиЕ 2. Для того чтобы әллипсы $\left(\mu x_{1}\right)^{2}+\left(h x_{1}+x_{2}\right)^{2}=C$ в случае $K_{1}$ были инвариантными кривыми уравнения (3), необходимо и достаточно, чтобы $g_{1}(t)=0, q_{1}(t)=-g_{2}^{2}(t) / 2$ с вероятностью 1 почти для всех $t \geqslant 0$ (т.е. в отличие от детерминированного случая при наличии трения $h \neq 0$ изображающая точка $M$ системы (3) с вероятностью 1 “диффундирует” по әллипсу). 
ЗАМЕчАНИЕ 1. Если вьполняются достаточные условия 1) теоремы 2 соответственно для $K_{i}$, то с вероятностью 1 для всех $t \geqslant 0$ вьполняются равенства

$$
\begin{gathered}
G_{i}(x(t))=G_{i}(x(0)) \exp \left\{I_{i}(t)\right\}, \quad i=1,2, \\
G_{3}(x(t))=G_{3}(x(0))+I_{3}(t),
\end{gathered}
$$

где $I_{i}(t)$ определены в $(6)$.

Поскольку в этих равенствах отсутствует стохастический интеграл Ито, то они удобны для управления с помощью функции $q_{i}(t)$ поведением $x(t)$ на фазовой плоскости. Например, для $K_{1}$ с вероятностью 1 для всех $t \geqslant 0$ имеют место оценки

$$
G_{1}(x(0)) e^{m} \leqslant G_{1}(x(t)) \leqslant G_{1}(x(0)) e^{M},
$$

где $m=\inf _{t \geqslant 0} I_{1}(t), M=\sup _{t \geqslant 0} I_{1}(t)$ и т. п.

Отметим, что система уравнений (3) совпадает с детерминированной системой (2) при $g_{i}(t) \equiv 0, i=1,2$, и условиях:

1) $q_{1}(t) \equiv-h, q_{2}(t) \equiv \sqrt{k^{2}-h^{2}}$ для $K_{1}$;

2) $q_{1}(t) \equiv \lambda_{1}, q_{2}(t) \equiv \lambda_{2}$ для $K_{2}$;

3) $q_{1}(t) \equiv-h, q_{2}(t) \equiv 1$ для $K_{3}$.

Кроме того, при $h>0$ детерминированная система (2) является абсолютно устойчивой, т. е. амплитуды колебаний $A_{i}(t)$ стремятся к нулю при $t \rightarrow \infty$ для $K_{i}, i=1,2,3$, при любых начальных отклонениях $u_{0}, \dot{u}_{0}$. При этом при $t \rightarrow \infty$ фазы колебаний в соответственных областях $D_{i}$ ведут себя следующим образом: $\varphi_{1}(t) \rightarrow-\infty$ (накручивание спирали на точку $(0,0)$ происходит по часовой стрелке), $\varphi_{2}(t) \rightarrow 0, \varphi_{3}(t) \rightarrow \pi / 2$.

При исследовании асимптотического поведения при $t \rightarrow \infty$ функций $A_{i}(t), \varphi_{i}(t)$ для уравнения (3) мы рассмотрим лишь случай $K_{1}$ (случаи $K_{2}, K_{3}$ исследуются аналогично [2]). Из явного вида решения уравнения (3) вытекает, что асимптотическое поведение $A_{1}(t), \varphi_{1}(t)$ зависит от асимптотического поведения величин

$$
\begin{gathered}
\beta_{i}(t)=\int_{0}^{t} g_{i}^{2}(s) d s, \quad i=1,2, \quad \alpha_{1}(t)=\int_{0}^{t}\left(q_{1}(s)+\frac{1}{2}\left[g_{2}^{2}(s)-g_{1}^{2}(s)\right]\right) d s, \\
\alpha_{2}(t)=\int_{0}^{t}\left[g_{1}(s) g_{2}(s)-q_{2}(s)\right] d s .
\end{gathered}
$$

Так, например, имеет место следующая теорема.

\section{TEOPEMA 3.}

1. Пусть $\lim _{t \rightarrow \infty} \beta_{1}(t)=\infty$. Если при этом

$m o \lim _{t \rightarrow \infty} A_{1}(t)=0 ;$ ecлu

$$
\varlimsup_{t \rightarrow \infty} \alpha_{1}(t)\left(2 \beta_{1}(t) \ln \ln \beta_{1}(t)\right)^{-1 / 2}<-1,
$$

$$
\varliminf_{t \rightarrow \infty} \alpha_{1}(t)\left(2 \beta_{1}(t) \ln \ln \beta_{1}(t)\right)^{-1 / 2}>1,
$$

mo $\lim _{t \rightarrow \infty} A_{1}(t)=\infty$.

2. Пусть $\lim _{t \rightarrow \infty} \beta_{2}(t)=\infty$. Если при этом

$$
\varlimsup_{t \rightarrow \infty} \alpha_{2}(t)\left(2 \beta_{2}(t) \ln \ln \beta_{2}(t)\right)^{-1 / 2}<-1,
$$

$m o \lim _{t \rightarrow \infty} \varphi_{1}(t)=-\infty ;$ ecлu

$$
\varliminf_{t \rightarrow \infty} \alpha_{2}(t)\left(2 \beta_{2}(t) \ln \ln \beta_{2}(t)\right)^{-1 / 2}>1,
$$

$m o \lim _{t \rightarrow \infty} \varphi_{1}(t)=\infty$. 
ДокАЗАТЕЛьСтво. Поскольку [9] имеет место представление

$$
\int_{0}^{t} g_{i}(s) d w(s)=\widetilde{w}_{i}\left(\beta_{i}(t)\right)
$$

где $\widetilde{w}_{i}(t)$ винеровские процессы, то, учитывая явный вид $A_{1}(t), \varphi_{1}(t)$, можем записать

$$
\begin{gathered}
A_{1}(t)=A_{1}(0) \exp \left\{\widehat{\beta}_{1}(t)\left[\frac{\alpha_{1}(t)}{\widehat{\beta}_{1}(t)}+\frac{\widetilde{w}_{1}\left(\beta_{1}(t)\right)}{\widehat{\beta}_{1}(t)}\right]\right\}, \\
\varphi_{1}(t)=\widehat{\beta}_{2}(t)\left[\frac{\varphi_{1}(0)}{\widehat{\beta}_{2}(t)}+\frac{\alpha_{2}(t)}{\widehat{\beta}_{2}(t)}-\frac{\widetilde{w}_{2}\left(\beta_{2}(t)\right)}{\widehat{\beta}_{2}(t)}\right],
\end{gathered}
$$

где

$$
\widehat{\beta}_{i}(t)=\left(2 \beta_{i}(t) \ln \ln \beta_{i}(t)\right)^{1 / 2} .
$$

Из этих представлений в силу “закона повторного логарифма", т.е.

$$
P\left\{\varlimsup_{t \rightarrow \infty} \frac{\left|\widetilde{w}_{i}\left(\beta_{i}(t)\right)\right|}{\widehat{\beta}_{i}(t)}=1\right\}=1, \quad i=1,2
$$

получаем доказательство теоремы 3.

Заметим, что исходя из явного вида решения $x(t)$ уравнения $(3)$, также удобно находить явньй вид коррелящионных функций $x(t)$.

\section{СПИСОК ЦИТИРОВАННОЙ ЛИТЕРАТУРЫ}

[1] Андронов А. А., Вит А. А., Хайкин С.Э. Теория колебаний. М.: Наука, 1981.

[2] Кулінич Г. Л.Якісний аналіз впливу на гармонічний осцилятор з тертям випадкових збурень типу "білого шуму" вздовж вектора фазовоі швидкості // Укр. матем. ж. 1997. Т. 49. № 1. C. $36-47$.

[3] Kulinich G. L. Qualitative analysis of the influence of random perturbations on the phase velocity of the harmonic oscillator // Random Oper. and Stoch. Equation. 1995. V. 3. № 2. P. 141-152.

[4] Скороход А. В. Асимптотические методы теории стохастических дифференциальных уравнений. Киев: Наукова думка, 1987.

[5] Гихман И.И., Скороход А. И. Теория случайных процессов. III. М.: Наука, 1975.

[6] Хасьминский Р. З. Устойчивость систем дифференциальных уравнений при случайных возмущениях их параметров. М.: Наука, 1969.

[7] Стратонович Р. Л. Избранные вопросы теории флуктуаций в радиотехнике. М.: Советское радио, 1961.

[8] Kulinich G. L. On the limiting behaviour of a harmonic oscillator with random external disturbance // J. Amer. Math. Soc. 1995. V. 8. №3. P. 265-274.

[9] Гихман И.И., Скороход А.В. Стохастические дифференциальные уравнения. Киев: Наукова думка, 1968.

[10] Бабчук В. Г., Кулинич Г. Л. Решение одного класса линейной системы стохастических дифференциальных уравнений Ито второго порядка с одним винеровским процессом // Теория вероятн. и ее примен. 1978. Т. 23. № 2. С. 457-458. 